\title{
Performance of Ultrawideband Wireless Tags for On-Body Radio Channel Characterisation
}

\author{
Mohammad Monirujjaman Khan, ${ }^{1,2}$ Qammer H. Abbasi, ${ }^{1}$ Akram Alomainy, ${ }^{1}$ and Yang Hao ${ }^{1}$ \\ ${ }^{1}$ School of Electronic Engineering and Computer Science, Queen Mary University of London, London E1 4NS, UK \\ ${ }^{2}$ Department of Electrical and Electronic Engineering, American International University-Bangladesh, Dhaka 1213, Bangladesh
}

Correspondence should be addressed to Mohammad Monirujjaman Khan, mohammad.khan@elec.qmul.ac.uk

Received 16 February 2012; Accepted 21 June 2012

Academic Editor: C. Aanandan

Copyright ( 2012 Mohammad Monirujjaman Khan et al. This is an open access article distributed under the Creative Commons Attribution License, which permits unrestricted use, distribution, and reproduction in any medium, provided the original work is properly cited.

\begin{abstract}
Experimental characterisation of on-body radio channel for ultrawideband (UWB) wireless active tags is reported in this paper. The aim of this study is to investigate the performance of the commercially available wireless tags on the UWB on-body radio channel characterisation. Measurement campaigns are performed in the chamber and in an indoor environment. Statistical path loss parameters of nine different on-body radio channels for static and dynamic cases are shown and analyzed. Results demonstrated that lognormal distribution provides the best fits for on-body propagation channels path loss model. The path loss was modeled as a function of distance for 34 different receiver locations for propagation along the front part of the body. A reduction of $11.46 \%$ path loss exponent is noticed in case of indoor environment as compared to anechoic chamber. In addition, path loss exponent is also extracted for different body parts (trunk, arms, and legs). Second-order channel parameters as fade probability (FP), level crossing rate (LCR), and average fade duration (AFD) are also investigated.
\end{abstract}

\section{Introduction}

Ultrawideband (UWB) communication is an exciting and innovative technology that has attracted much attention and experienced considerable growth in the past few years due to its distinctive characteristics. It is a low-power, high data rate technology that minimizes multipath interference due to late time-of-arrival. Its low-power requirement due to control over duty cycle allows longer battery life and also introduces green radio system. One of the most potential areas of UWB applications is the body-centric wireless networks where various units/sensors are scattered on/around the human body to measure specified physiological data that is, patient monitoring for healthcare applications [1-3].

In the past few years researchers have been thoroughly investigating narrowband and ultrawideband on-body radio channels. In [4-7], on-body radio channel characterisation was presented at the unlicensed frequency band of $2.45 \mathrm{GHz}$. UWB on-body radio channel characterisation and system level modelling for body-centric wireless networks have been presented extensively in the open literature $[2,8-20]$.
In [8-20], UWB on-body propagation channels have been characterized, and their behaviour has been investigated in indoor and chamber for standstill, various postured and dynamic human body based on different antennas. Most UWB on-body channel measurements are performed using two standalone antennas and cables connecting to a vector network or spectrum analyzer which is more a controlled environment and restrictive; however, in real life scenarios potential UWB body-centric wireless network needs to be integrated with compact sensors and provides efficient and reliable communication channels. Critical issues remain with regards to indoor propagations, radio channel characterization, and human body effect which they need to be addressed before the concept can be deployed for commercial applications.

In this paper, measurement campaigns were performed in the chamber and indoor environment using commercially available UWB wearable active tags and reader. The main aim of this study is to investigate the performance of the commercially available wireless tags on the UWB on-body radio channel characterization. Nine different on-body radio 
channels are investigated and the effects of the body movements on the path loss are analysed. Second-order statistics for three different on-body links are investigated. The results reported here provide information on optimum sensor locations on the body considering efficient and reliable communication links for various applications, for example, healthcare and performance monitoring.

The rest of the paper is organised as follows: Section 2 illustrates the measurement settings, and it briefly introduces the UWB tags, Sections 3 and 4 present the measurement results and on-body radio channel parameters and modelling aspects, and finally Section 5 draws the main conclusion of the presented study.

\section{Measurement Settings}

Measurement campaigns were performed using UWB wearable active tags and reader provided by time domain PLUS [21]. For this measurement purpose, a real human subject was used. The test subject was an adult male of mass $90 \mathrm{Kg}$, height 1.68 meter, and chest circumference $114 \mathrm{~cm}$. Nine different ultrawideband wireless active transmitter tags were attached at different locations on the human body: left/right chest, left/right thigh, left wrist, left/right ankle, left elbow, and left ear, as shown in Figure 1, while the UWB antenna connected with the reader was placed on the left waist of the human subject for tag's signal reception. Two measurement scenarios are considered: static and dynamic human body. For static case, subject was standing still for a period of 60 seconds wearing nine tags on the body and, for the movement case, the subject was walking 5 steps ahead and 5 steps back, starting with the left leg and right arm as a normal walking speed. For the dynamic case, the measurement duration was again 60 seconds while the subject was doing the same walking movement for the measurement duration. Location-based software was used to save the tags transmission ID, received signal strength (RSSI), and time of arrival data from the reader. The UWB tags are battery powered, and the duration of the battery life is four years since the tags only transmit UWB pulses every one second. The tag's transmit power is $-13.01 \mathrm{dBm}$ which is around $40 \mathrm{~dB}$ less than mobile phone transmit power. The operating frequency of the tags used for this measurement is $5.9 \sim 7.25 \mathrm{GHz}$ with a centre frequency of $6.6 \mathrm{GHz}$. The UWB tag is small and durable, with a plastic housing that allows it to be attached to assets or people. The dimension of the tag is $(13 \mathrm{~mm} \times 36 \mathrm{~mm} \times 33 \mathrm{~mm})$ and the weight is $0.74 \mathrm{oz}(22 \mathrm{~g})$. Figures 2(a), 2(b), and 2(c) show the UWB tag encased inside the plastic housing, the bottom view of the tag without plastic housing, and the top view of the tag showing the tag antenna [21]. The free space and on-body radiation patterns of the tag antenna at $6.6 \mathrm{GHz}$ are measured in the anechoic chamber. The on-body radiation patterns are measured by placing the tag antenna on the same test subject as mentioned earlier. Figures 3(a) and 3(b) show comparison of free space and on-body radiation patterns ( $X Y$ and $Y Z$ planes) at $6.6 \mathrm{GHz}$ of the tag antenna. The tag antenna has monopolelike radiation patterns. When the tag is mounted on the body, the radiation pattern is omnidirectional over the surface $(X Z$

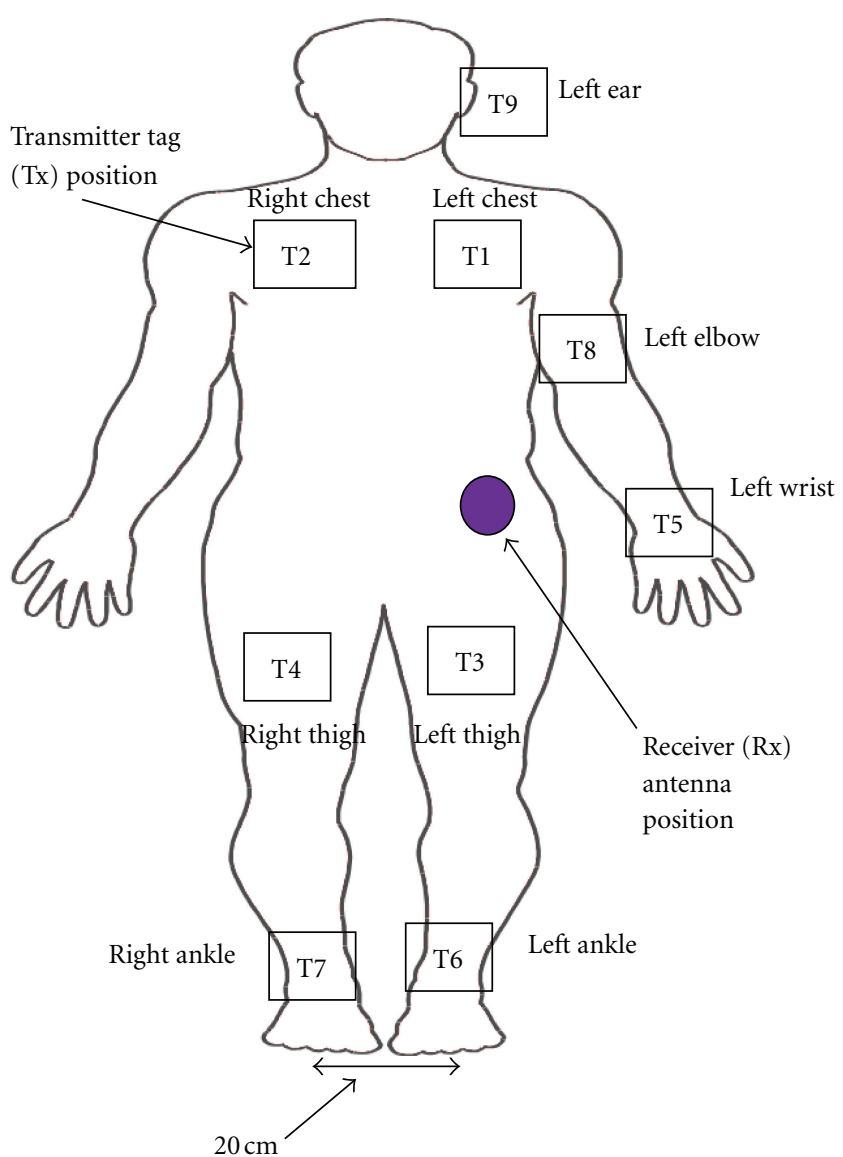

FIGURE 1: On-body measurement settings showing the receiver antenna is on the left waist, and nine transmitter tags are on different locations of the body (nine static and dynamic channels cases analysed).

plane) of the body (test subject) and the elevation plane ( $Y Z$ ) radiation pattern is directed towards off the body direction with a null in the main beam.

The measurement was first performed in the anechoic chamber to eliminate multipath reflections from surrounding environment and then repeated in the Body-Centric Wireless Sensor Lab at Queen Mary University of London to consider the effect of the indoor environment on the onbody radio propagation channels. Figure 4 shows the dimensions and geometry of the Body-Centric Wireless Sensor Lab. The total area of the lab is $45 \mathrm{~m}^{2}$ which includes a meeting area, treadmill machine, workstations, and a hospital bed for healthcare applications. The measured Received Signal Strength Indicator (RSSI) level for each transmitter tag is recorded over the measurement duration of 60 seconds for each different location.

\section{UWB On-Body Radio Channel Parameters}

3.1. On-Body Radio Channel Characterisation. In this work, the path loss for nine different on-body channels was calculated from the measured RSSI for each transmitter tag. The cumulative distribution function (CDF) of the path loss 


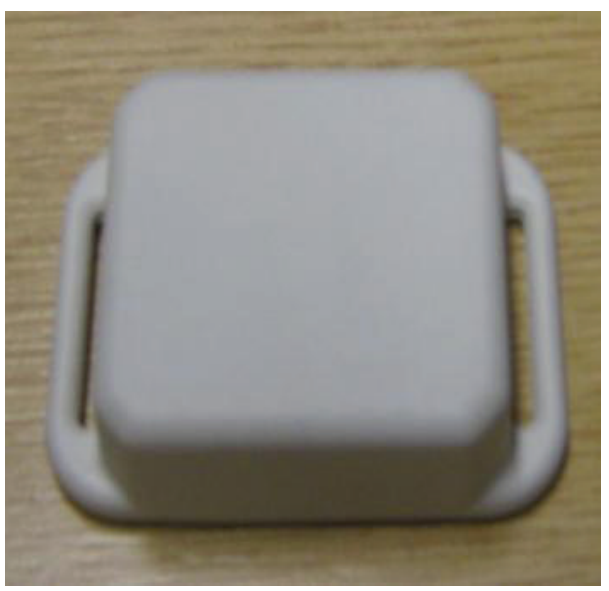

(a)

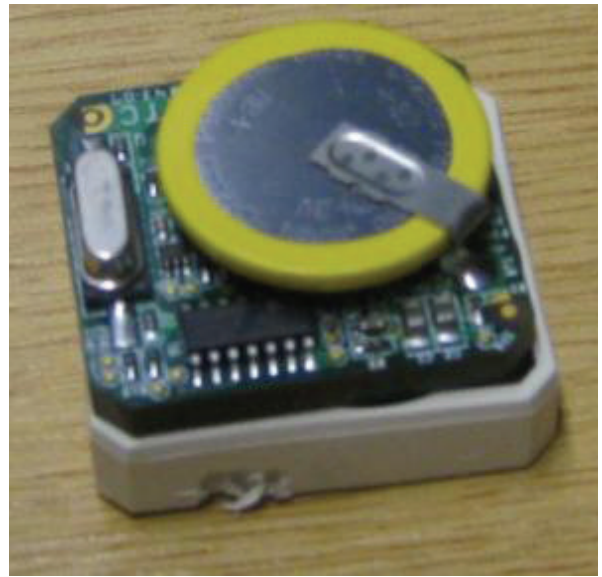

(b)

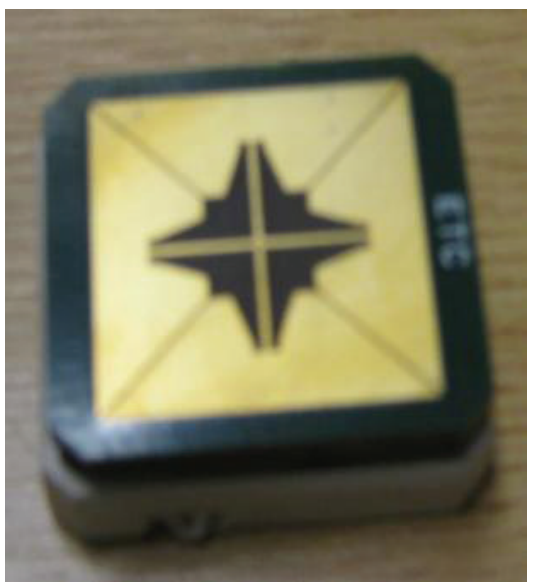

(c)

FIGURE 2: (a) UWB active transmitter tag encased inside the plastic housing, (b) tag without plastic housing and bottom view, and (c) top view of the tag showing the transmitter tag antenna.

variations both in the chamber and indoor environment for static and dynamic scenarios of nine different on-body radio channels is compared to well-known distributions as Normal, Lognormal, Nakagami, Rayleigh, Weibull, Gamma, and Rician adopting the Akaike criteria and on the basis of the tested results, lognormal distribution provides the best fits to these measured results (Figure 5). The Akaike information criteria is a method widely used to evaluate the goodness of a statistical fit $[3,22]$. The second-order AIC $\left(\mathrm{AIC}_{c}\right)$ is defined as

$$
\mathrm{AIC}_{c}=-2 \ln (L)+2 k+\frac{2 k(k+1)}{n-K-1},
$$

where $L$ is the maximised likelihood, $K$ is the number of parameters estimated for that distribution, and $n$ is the number of samples of the experiment. The seven distributions mentioned above are all two parameter distributions $(K=$ 2 ) except the Rayleigh $(K=1)$. In this measurement, the sample size is $(n=60)$.

The maximised log likelihood has been obtained from the MATLAB estimates. The Akaike information criteria can be used as a relative measure such that the model with the lowest AIC means better statistical model and the criterion is used to classify models from the best to worse; to facilitate this process, the relative AIC is considered and results are normalized to the lowest value obtained

$$
\Delta_{i}=\mathrm{AIC}_{c}-\min \left(\mathrm{AIC}_{c}\right)
$$

A zero value indicates the best fitness. Comparison of different distributions adopting AKAI information criteria is shown in Table 1.

Figures 6 and 7 show a comparison of the measured average path loss $(\mu)$ and standard deviation $(\sigma)$ of the fitted lognormal distribution that are applied to model the path loss variations for the nine on-body radio channels, for the static and walking scenarios, respectively.

In the chamber, for both standing and walking cases, the highest path loss is noticed for the receiver to leftear link, while the lowest is the receiver to left-thigh link (Figure 6). For the reader to left-ear link the communication distance between the reader antenna and the transmitter tag is larger; in addition, due to the different orientation of the tag located on the left ear, non-line-of-sight (NLOS) 


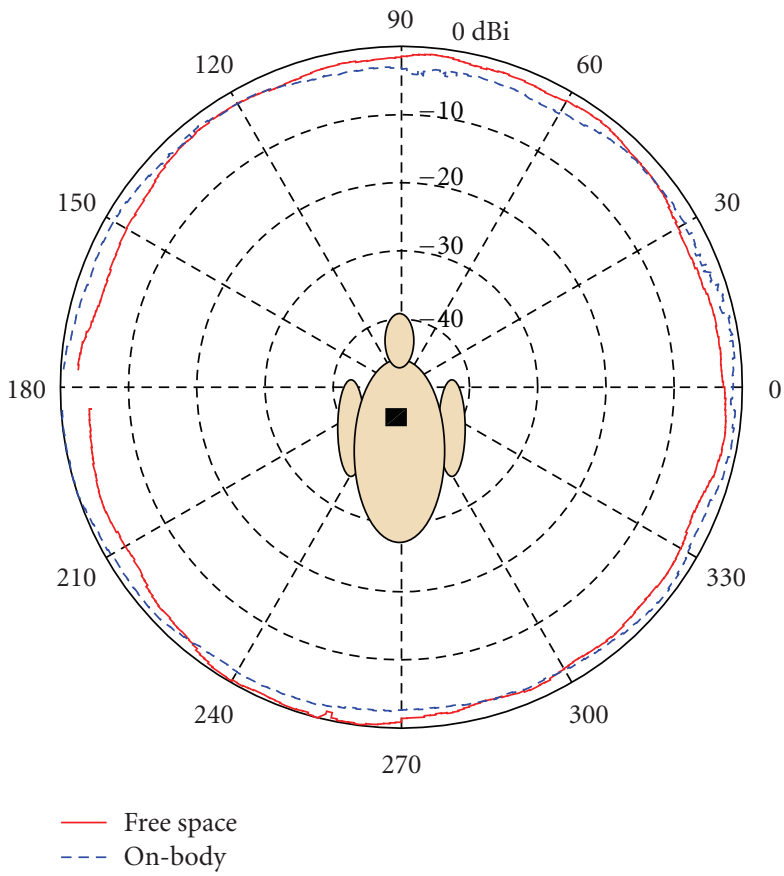

(a) $X Z$ plane

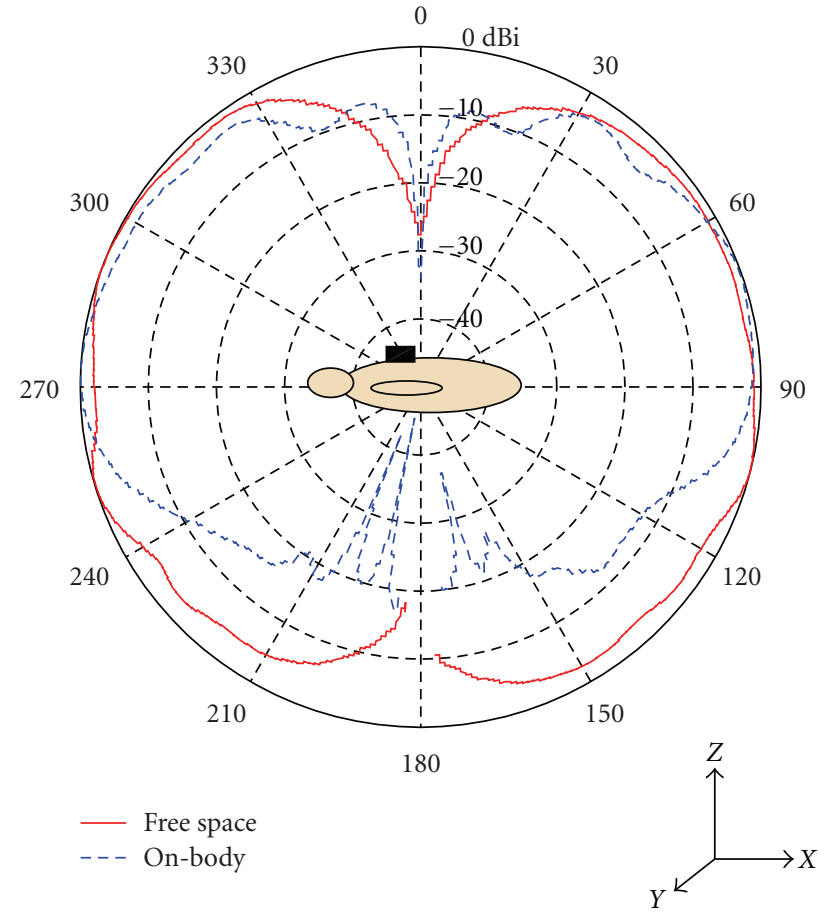

(b) YZ plane

Figure 3: Measured free space and on-body radiation patterns at $6.6 \mathrm{GHz}$ of the tags (a) $X Z$ plane and (b) $Y Z$ plane.

TABle 1: Comparison of different distributions adopting AKAI information criteria for nine on-body links measured in the chamber and indoor.

\begin{tabular}{lcccccccc}
\hline Tag position & Scenario & Normal & Lognormal & Gamma & Nakagami & Rayleigh & Rician & Weibull \\
\hline \multirow{2}{*}{ L. chest } & Chamber & 0.286 & 0 & 0.080 & 0.176 & 324.69 & 0.276 & 11.12 \\
& Indoor & 0.892 & 0 & 0.282 & 0.282 & 313.88 & 0.884 & 16.74 \\
R. chest & Chamber & 0.010 & 0.078 & 0.040 & 0.016 & 323.22 & 0 & 5.268 \\
& Indoor & 0.398 & 0.390 & 0 & 0.190 & 296.31 & 0.390 & 12.68 \\
L. thigh & Chamber & 1.490 & 0 & 0.474 & 0.970 & 292.15 & 1.48 & 24.48 \\
& Indoor & 0.474 & 0 & 0.140 & 0.2980 & 310.63 & 0.470 & 16.40 \\
R. thigh & Chamber & 0.994 & 1.102 & 1.312 & 1.142 & 249.58 & 0.984 & 0 \\
& Indoor & 0.008 & 0.420 & 0.260 & 0.130 & 290.26 & 0 & 5.450 \\
L. wrist & Chamber & 0.530 & 0 & 0.120 & 0.290 & 203.40 & 0.520 & 8.530 \\
& Indoor & 0.022 & 0.160 & 0.060 & 0 & 188.38 & 0.012 & 1.700 \\
L. ankle & Chamber & 0.830 & 0 & 0.240 & 0.520 & 264.01 & 0.820 & 15.25 \\
& Indoor & 2.010 & 0 & 0.63 & 1.30 & 234.93 & 2.00 \\
R. ankle & Chamber & 0.490 & 0 & 0.110 & 0.260 & 220.40 & 0.480 \\
& Indoor & 0.290 & 0 & 0.056 & 0.142 & 155.34 & 0.280 & 9.160 \\
L. elbow & Chamber & 0.820 & 0 & 0.250 & 0.514 & 241.55 & 0.812 \\
& Indoor & 0.086 & 0.014 & 0 & 0.014 & 219.99 & 0.076 \\
L. ear & Chamber & 0.010 & 0 & 0.050 & 0.0220 & 288.55 & 0.096 \\
\hline
\end{tabular}

communications exist, which cause the highest path loss value for this channel. For this case (the receiver to left-ear link) due to different orientation of the tag located on the left ear, the polarization mismatch occurs between the tag and the reader which also causes the higher path loss value for this link. For the left-thigh link, there are a clear line-ofsight (LOS) communication and the lowest communication distance between the reader and the transmitter tag.

In the indoor environment due to reflecting area and contributions of multipath reflection the right chest and 


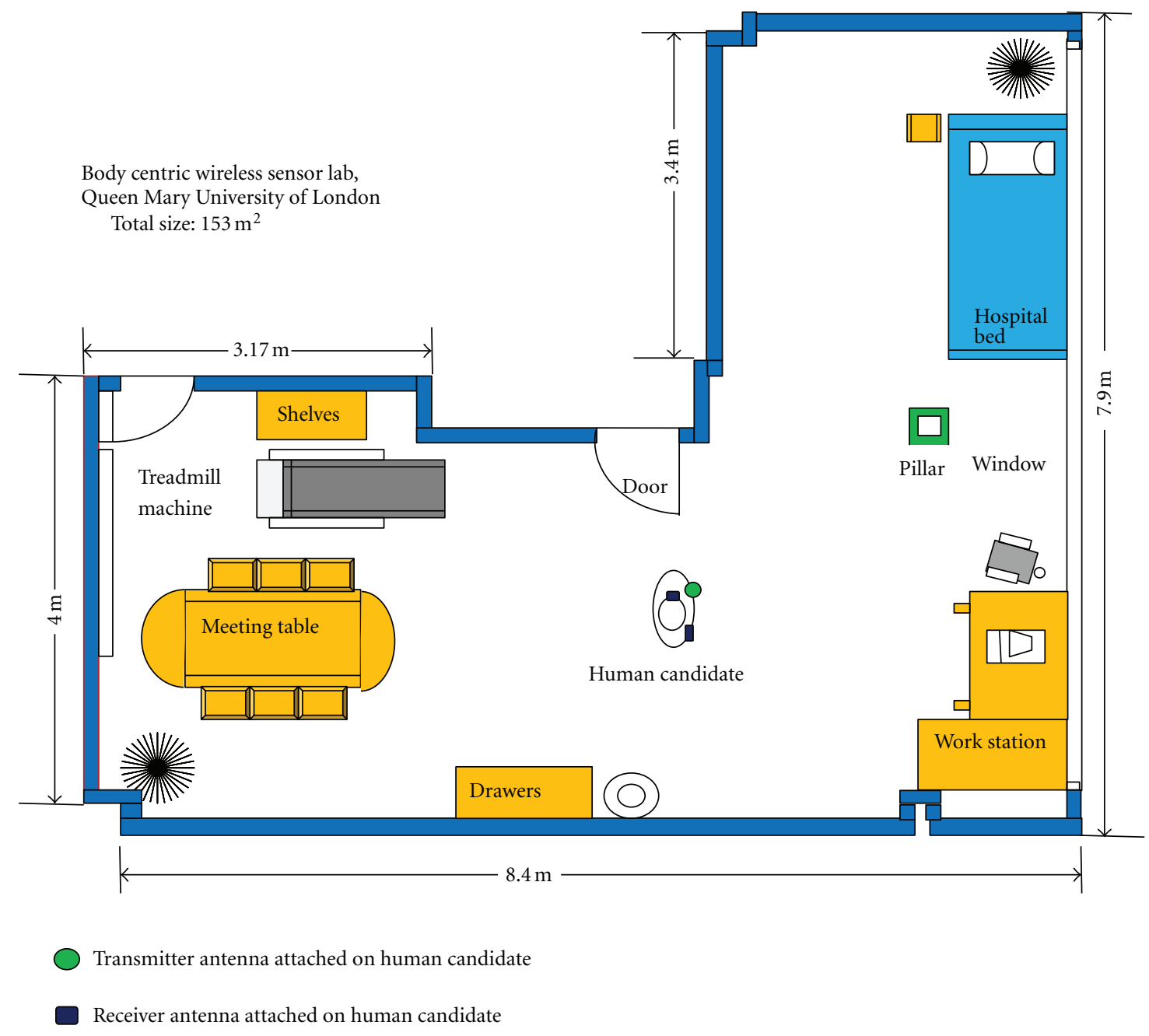

Figure 4: Dimensions and geometry of the Body-Centric Wireless Sensor Lab (housed within the Department of Electronic engineering, Queen Mary University of London, London, UK) where the indoor on-body radio propagation measurements for the presented work are performed.

left-ear (for static case) and right thigh and chest channels (for movement cases) experience the highest path loss value, while the left thigh channel experiences the lowest. Most of the on-body channels experience higher path loss value when measurements are made in the chamber, due to the nonreflecting environment. The average path loss of all nine channels in the chamber, for static and walking cases, is $81.44 \mathrm{~dB}$ and $80.68 \mathrm{~dB}$, whereas $79.22 \mathrm{~dB}$ and $80.00 \mathrm{~dB}$ are found in the indoor environment, respectively.

The variation of the path loss for the nine different onbody channels is also compared for standing and walking cases, to study the trend of the changes of path loss for each channel in these two different scenarios. For the two different scenarios (i.e., standing and walking) a maximum of $8.23 \mathrm{~dB}$ and $6.88 \mathrm{~dB}$ variation of average path loss of a channel is noticed, which occurred for the left wrist channel both in indoor environment and chamber, respectively (Figure 6). During walking scenarios, the tag located on the wrist moves between LOS and NLOS communications scenarios, and the communication distance between the receiver and the transmitter is also changed greatly, causing the path loss data to vary the most with respect to the standing case for this channel.

The highest standard deviation $(\sigma)$ value for the dynamic case is noticed for the left wrist and right ankle channels, which are considered the least stable (data spread the most from the average path loss) channels, whereas the lowest is noticed for the left thigh and chest channels; these channels are considered the most stable (see Figure 7). Movement of the human body has the highest effect on the wrist and ankle channels and the least on chest and left thigh channels. In comparison to the chamber, the standard deviation value is found to be higher in the indoor environment due to the effects from the indoor reflecting multipath environment.

3.2. Path Loss versus Distance. Measurements were also performed in order to investigate the propagation along the front part of the whole body; see Figure 8. The same test subject was used for this case as was used for the previous case. The receiver antenna connected to the reader was placed 


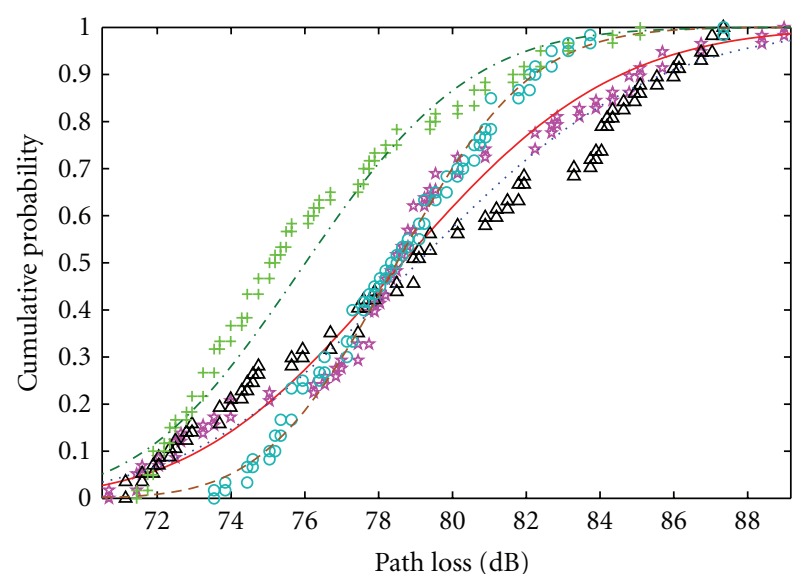

* Left wrist chamber

$\Delta$ Left wrist sensor lab

- Left ankle chamber

+ Left ankle sensor lab

Figure 5: Cumulative distribution function of the left wrist and left ankle on-body radio channels when subject was walking measured in the chamber and in indoor environment.

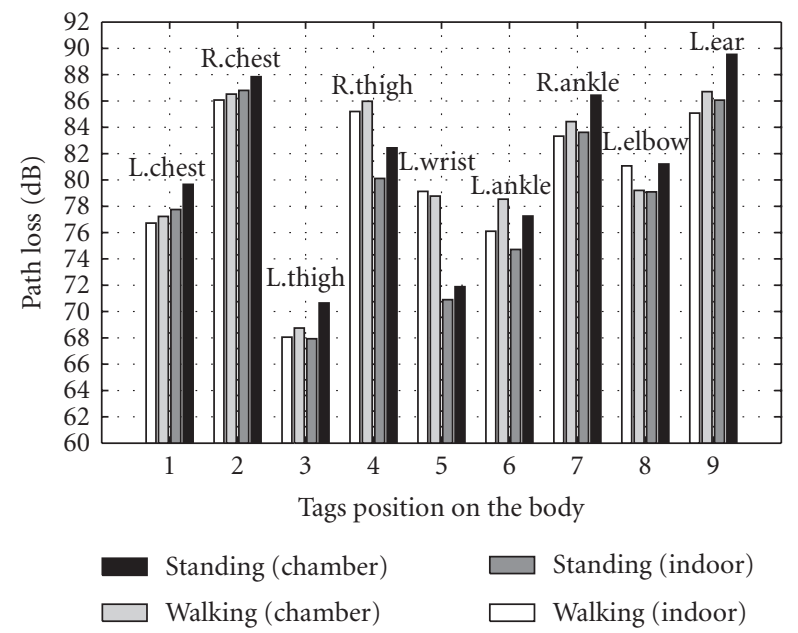

FIGURE 6: Comparison of average path loss of nine UWB on-body radio channels for standing and walking scenarios measured in the chamber and in indoor.

TABle 2: Path loss parameters for whole body and different body parts.

\begin{tabular}{lcccccc}
\hline \multirow{2}{*}{ Body parts } & \multicolumn{3}{c}{ Channels } & \multicolumn{3}{c}{ Chamber } \\
& $\gamma$ & $\mathrm{PL}_{\mathrm{dB}}\left(d_{0}\right)$ & $\sigma$ & $\gamma$ & $\mathrm{PL}_{\mathrm{dB}}\left(d_{0}\right)$ & $\sigma$ \\
\hline All body & 2.53 & 68 & 6.01 & 2.24 & 67 & 5.11 \\
Trunk & 3.48 & 66 & 2.07 & 2.85 & 65.8 & 2.12 \\
Arms & 3.46 & 64 & 4.35 & 2.84 & 65.7 & 2.19 \\
Legs & 2.89 & 58.3 & 5.46 & 2.66 & 59 & 6.47 \\
\hline
\end{tabular}

on the left waist, while the transmitter tags were placed on 34 different locations on the front part of the body (trunk,

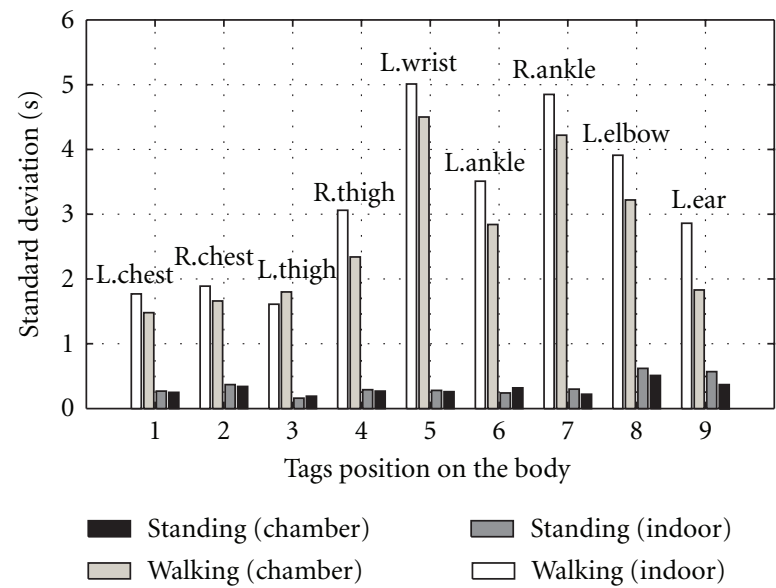

FiguRe 7: Comparison of standard deviation $(\sigma)$ of nine different on-body radio channels for standing and walking scenarios measured in the chamber and indoor.

arms, legs, and head). In this case, during the measurement, the subject was standing still for 60 seconds, and, the data were saved for that period of time for each transmitter tag location.

It is well known that the average received signal decreases logarithmically with distance (for both indoor and outdoor environments).

The path loss can be modeled as a linear function of the logarithmic distance between transmitter and receiver as explained in [23],

$$
\mathrm{PL}_{\mathrm{dB}}(d)=\mathrm{PL}_{\mathrm{dB}}\left(d_{0}\right)+10 \gamma \log \left(\frac{d}{d_{0}}\right)+X_{\sigma},
$$

where $d$ is the distance between transmitter and receiver, $d_{0}$ is a reference distance set in measurement (in this study it is set to $10 \mathrm{~cm}), \mathrm{PL}_{\mathrm{dB}}\left(d_{0}\right)$ is the path loss value at the reference distance, and $X_{\sigma}$ is the shadowing fading. The parameter $\gamma$ is the path loss exponent that indicates the rate at which the path loss increases with distance. This parameter $\gamma$ depends on the structure of environment (chamber or indoor) but also on the type of trajectory (trunk or legs).

A least-square fit technique was performed on the measured path loss for the 34 different transmitter locations, (Figure 8) to extract the path loss exponent. Figure 9 shows the measured value and modelled path loss for on-body channels versus logarithmic Tx-Rx separation distance. The path loss exponent was found to be 2.52 in the chamber and 2.24 in indoor (Table 2). In the indoor environment, the path loss exponent was found to be lower. When measurements are performed indoors, the reflections from surroundings scatters increase the received power, causing reduction in the path loss exponent. A reduction of $11.46 \%$ was noticed in indoor compared to the chamber.

$X_{\sigma}$ is a zero mean, normal distributed statistical variable, and is introduced to consider the deviation of the measurements from the calculated average path loss. Figure 10 shows the deviation of measurements from the average path loss fitted to a normal distribution for both measurement cases. 


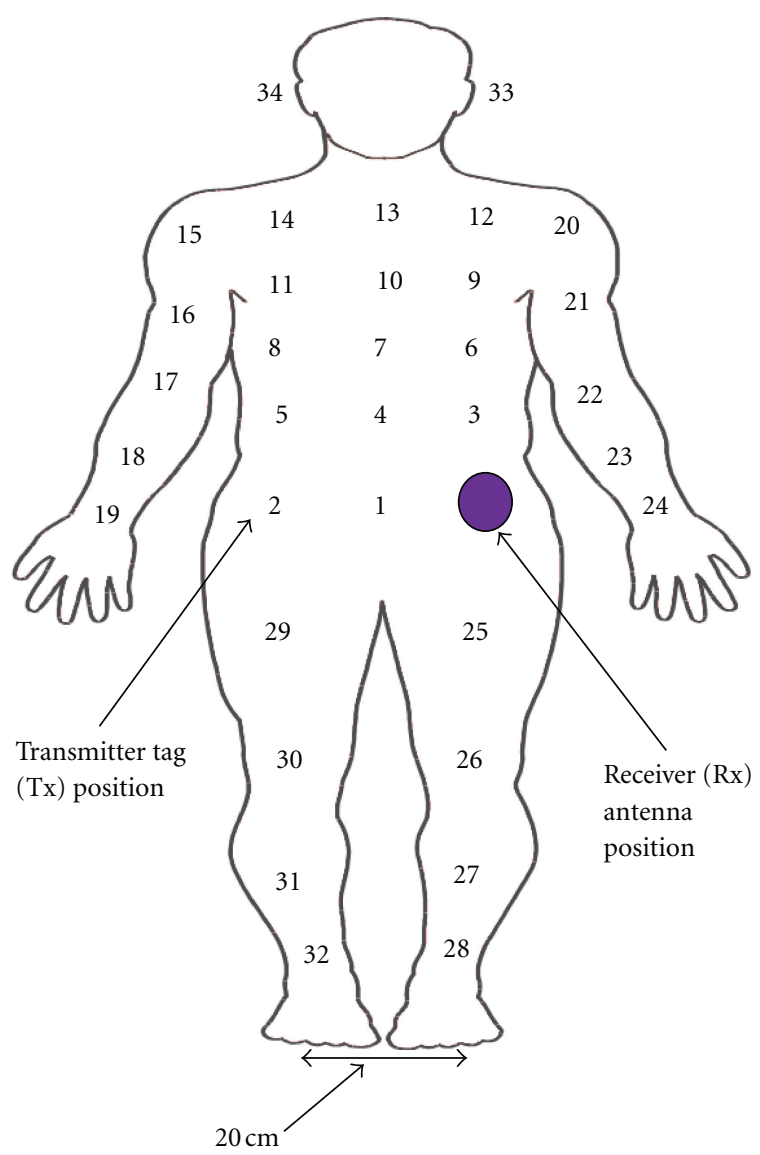

Figure 8: Location of the receiver antenna and positions of the 34 transmitter tags on the human subject.

In this case, the standard deviation of the normal distribution is found to be lower in the indoor environment.

The path loss exponent for propagation along different body parts, including trunk, arms, and legs, was extracted for both measurement cases and is summarised in Table 2 . Different body parts show different path loss exponent values. The lowest value is found for the legs (2.89), whereas the highest was found for the trunk (3.48). For the trunk, the propagation mechanism is mainly creeping waves, which experience higher signal attenuation, resulting in higher exponent value [19]. In addition, in the trunk, there are higher diffraction and reflection from the human body parts that also increase the path loss exponent. For the arms case, the path loss exponent is higher, as some of the tags located on the arms support NLOS communications. For the legs, the propagation mechanism is mainly free space waves, guided waves and line-of-sight (LOS), which experience lower signal attenuation, resulting in lower path loss exponent. The lowest mean path loss at the reference distance is found for the leg parts in both measurement cases.

\section{Second-Order Statistics for Different On-Body Links}

The level crossing rate (LCR), average fade duration (AFD), and probability of fade $(\mathrm{PF})$ are commonly applied in radio

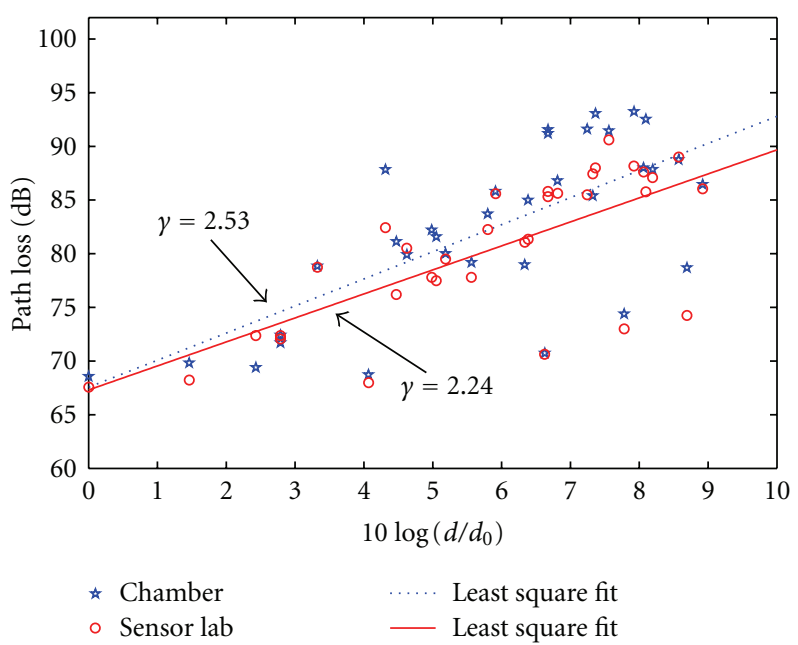

FIGURE 9: Measured and modelled path loss for on-body channel versus logarithmic Tx-Rx separation distance for UWB tags measured in the chamber and in indoor environments.

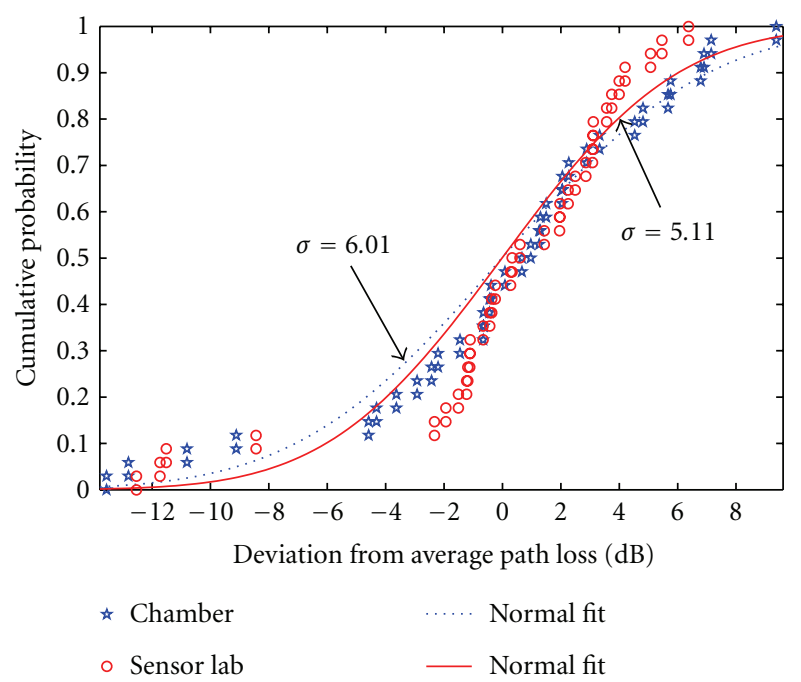

Figure 10: Deviation of the measurements from the average path loss fitted to normal distribution for both chamber and indoor measurement scenarios.

channel analysis to describe and investigate the severity of fading [24]. In this study, the second-order statistics are applied on the variation of RSSI due to the movement caused by walking over the measurement period (as shown in Figures 11(a) and 11(b)). Three different on-body radio links have been considered, namely, left waist to left wrist, left waist to left ankle, and left waist to left ear.

4.1. Fade Probability (FP). The probability of fade is the probability that a fading signal remains below the fade level or certain threshold level [24]. Figure 12 shows the comparison of fading probability for left wrist, left ankle, and left ear on-body channels for the walking case, measured in the chamber and in indoor. The fade levels shown in Figure 12 are the RSSI values for walking and normalized 


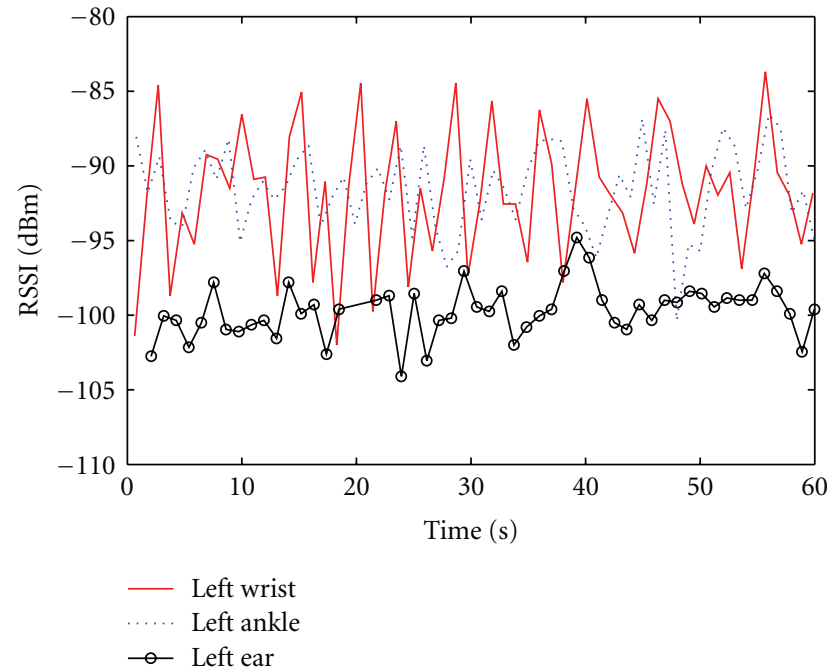

(a) Chamber

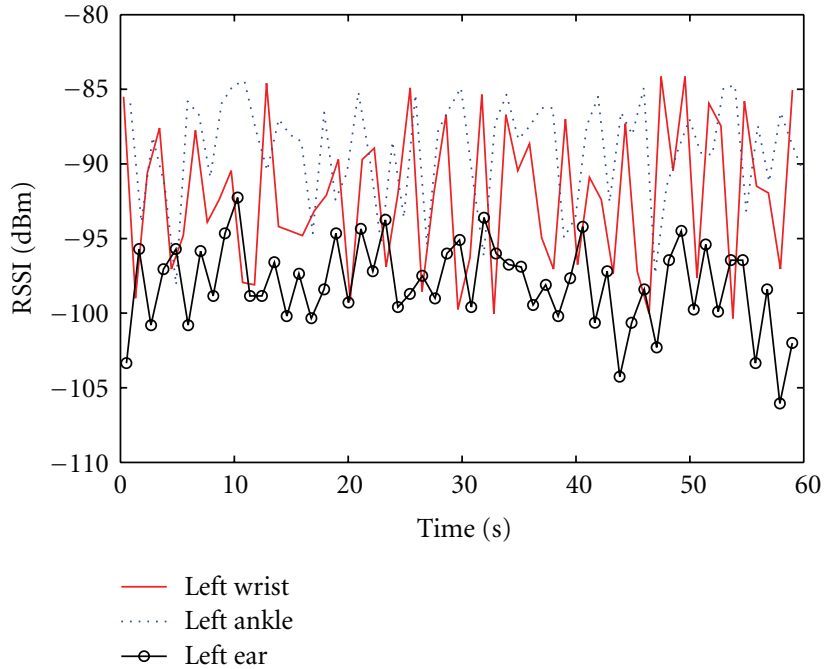

(b) Indoor

FIGURE 11: Comparison of RSSI level for three different on-body channels as left wrist, left ankle, and left ear when human subject was walking 5 steps ahead and 5 steps backward measured in the (a) chamber, (b) indoor.

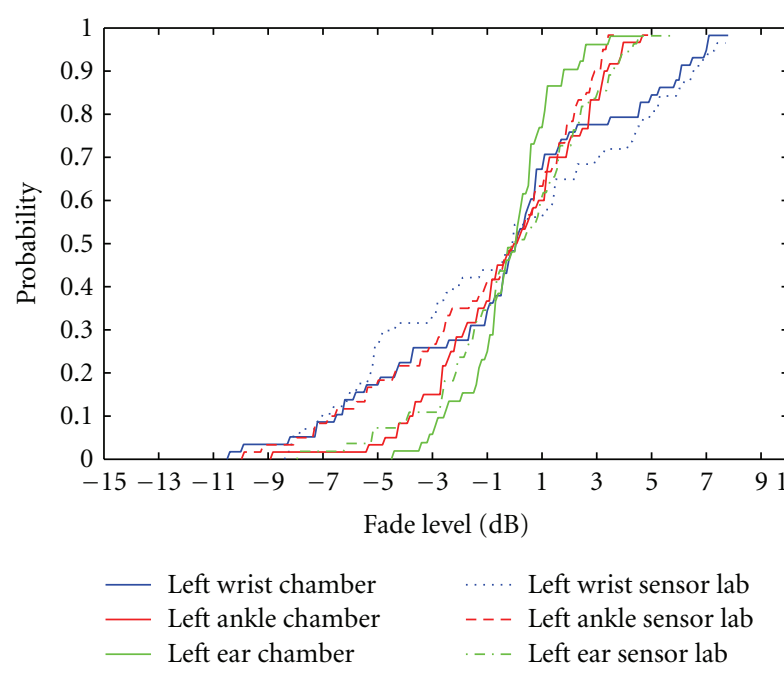

Figure 12: Comparison of fading probability (FP) for three different on-body channels as left wrist, left ankle, and left ear when subject was walking 5 steps ahead and back measured in the chamber and in indoor environment.

by the corresponding medians, and for the total fade level the increment of $0.01 \mathrm{~dB}$ is considered. The maximum fade level is noticed for the wrist and ankle channels, whereas the lowest is at the ear link. Comparing the two environments, the fade level is higher indoors for the left ear and ankle channels but lower for the wrist channel, which can be the effects of the multipath scattering environment in indoor. The fading probability at $-3 \mathrm{~dB}$ fade level is found to be higher for all three different on-body links in indoor. At $-3 \mathrm{~dB}$ fade depth, the fade probability of these three channels is between $7 \%$ and $27 \%$ in the chamber, while in indoor, it

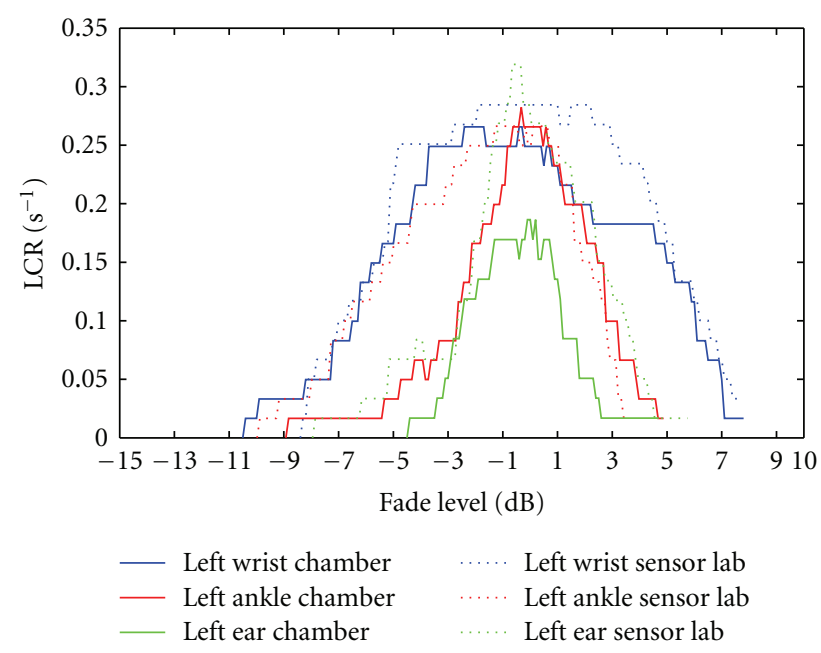

FIGURE 13: Comparison of level crossing rate (LCR) for three onbody channels as left wrist, left ankle, and left ear when subject was walking 5 steps ahead and back measured in the chamber and in indoor environment.

is between $12 \%$ and $33 \%$. Out of these three channels, the fade probability at $-3 \mathrm{~dB}$ is noticed higher for the left wrist channel, with a value of $33 \%$ measured in indoor, while the lowest is for the left ear channel, with a value of $7 \%$ measured in the chamber. Changing measurement environment does not change the fade probability for left ear link at $-3 \mathrm{~dB}$ fade depth but which has higher effects for ankle and wrist channels.

4.2. Level Crossing Rate (LCR). The level crossing rate (LCR) for a signal is the number of crossings of the signal with respect to a given threshold or specified fade level in the 


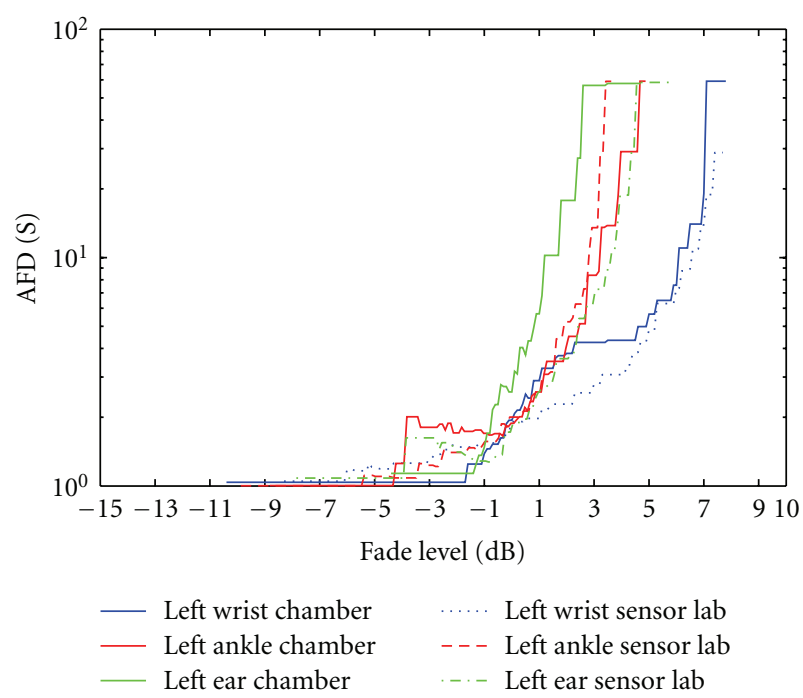

FIGURE 14: Comparison of average fade duration for three on-body channels as left wrist, left ankle, and left ear when subject was walking measured in the chamber and indoor environment.

positive going direction in a unit of time [24]. Figure 13 shows a comparison of the level crossing rate for the three considered links when the subject was walking. At a specified fade depth of $-3 \mathrm{~dB}$ for the walking case, the LCR for these three on-body channels is mostly found to be higher in indoor. At $-3 \mathrm{~dB}$ fade depth, the LCR in the chamber for these three channels is in the range of $0.05 \mathrm{~s}^{-1}-0.25$ $\mathrm{s}^{-1}$ while it is $0.07 \mathrm{~s}^{-1}-28 \mathrm{~s}^{-1}$ in the indoor environments. Both in the chamber and indoor environments, the highest LCR value at $-3 \mathrm{~dB}$ fade depth is noticed for wrist channel whereas the lowest is for ear channel. The LCR for the ankle link varies greatly in between the chamber and the indoor environments.

4.3. Average Fade Duration (AFD). The average fade duration is the average duration of time during which the fading signal remains below the specified fade level [24]. Figure 14 shows comparison of average fade duration for three different on-body channels, that is, as wrist, ankle, and ear measured in the chamber and in indoor for walking human subject. The AFDs characteristically increase with decreasing of fade depth.

For walking case, the left ankle channel has the highest AFD with the value of 1.9 seconds at the fade depth of $-3 \mathrm{~dB}$ in the chamber. At $-3 \mathrm{~dB}$ fade depth, the AFD is higher in the indoor environment for wrist and ear channels, while lower for the ankle. The AFD for all three channels is in the range of 1.1-1.9 seconds.

\section{Conclusion}

UWB on-body radio propagation channel measurements were performed using ultrawideband (UWB) wireless tags and reader in the chamber and indoor environments. Nine different UWB on-body radio channels were investigated for static and movement scenarios. Results demonstrated that lognormal distribution provides the best fits for on-body propagation channels path loss model. In this study, left thigh link shows the lowest path loss, whereas the left ear and right chest show the highest. The study shows that due to different scenarios (i.e., standing and walking) an onbody link experiences up to $8.23 \mathrm{~dB}$ variations in path loss. Path loss exponent for individual body parts is extracted, where the lowest path loss exponent and the path loss at the reference distance are noticed for the legs part. Second-order channel parameters as (LCR), (FP), and (AFD) for three onbody links are investigated. Results and analysis showed that at a specified fade depth of $-3 \mathrm{~dB}$, the fade probability, level crossing rate, and average fade duration are found mostly higher in the indoor as compared to chamber.

\section{Acknowledgments}

The authors would like to thank John Dupuy and Sanjoy Mazumdar for their help and assistance with the measurements. Many thanks to Yuri Nechayev (University of Birmingham) for his fruitful discussions.

\section{References}

[1] P. S. Hall and Y. Hao, Antennas and Propagation For BodyCentric Wireless Communications, Artech House, Norwood, Mass, USA, 2006.

[2] J. Foerester, E. Green, S. Somayazulu, and D. Leeper, "Ultrawideband for short- or medium-range wireless communications," Intel Technology Journal, vol. Q2, 2001.

[3] A. Fort, C. Desset, P. de Doncker, P. Wambacq, and L. van Biesen, "An ultra-wideband body area propagation channel model — from statistics to implementation," IEEE Transactions on Microwave Theory and Techniques, vol. 54, no. 4, pp. 18201826, 2006.

[4] Z. H. Hu, Y. I. Nechayev, P. S. Hall, C. C. Constantinou, and Y. Hao, "Measurements and statistical analysis of on-body channel fading at $2.45 \mathrm{GHz}$," IEEE Antennas and Wireless Propagation Letters, vol. 6, pp. 612-615, 2007.

[5] A. Alomainy, Y. Hao, A. Owadally et al., "Statistical analysis and performance evaluation for on-body radio propagation with microstrip patch antennas," IEEE Transactions on Antennas and Propagation, vol. 55, no. 1, pp. 245-248, 2007.

[6] S. L. Cotton, G. A. Conway, and W. G. Scanlon, "A timedomain approach to the analysis and modeling of on-body propagation characteristics using synchronized measurements at $2.45 \mathrm{GHz}$," IEEE Transactions on Antennas and Propagation, vol. 57, no. 4, pp. 943-955, 2009.

[7] Y. I. Nechayev, Z. H. Hu, and P. S. Hall, "Short-term and longterm fading of on-body transmission channels at $2.45 \mathrm{GHz}$," in Proceedings of the Loughborough Antennas and Propagation Conference (LAPC'09), pp. 657-660, Loughborough, UK, November 2009.

[8] A. Fort, C. Desset, J. Ryckaert, P. de Doncker, L. van Biesen, and P. Wambacq, "Characterization of the ultra wideband body area propagation channel," in Proceedings of the IEEE International Conference on Ultra-Wideband (ICU'05), pp. 2227, September 2005.

[9] A. Alomainy, Y. Hao, X. Hu, C. G. Parini, and P. S. Hall, "UWB on-body radio propagation and system modelling for wireless 
body-centric networks," IEE Proceedings: Communications, vol. 153, no. 1, pp. 107-114, 2006.

[10] Q. H. Abbasi, A. Sani, A. Alomainy, and Y. Hao, "On-body radio channel characterization and system-level modeling for multiband OFDM ultra-wideband body-centric wireless network," IEEE Transactions on Microwave Theory and Techniques, vol. 58, no. 12, pp. 3485-3492, 2010.

[11] Q. Wang and J. Wang, "Performance of on-body chest-towaist UWB communication link," IEEE Microwave and Wireless Components Letters, vol. 19, no. 2, pp. 119-121, 2009.

[12] Q. Wang, T. Tayamachi, I. Kimura, and J. Wang, "An on-body channel model for UWB body area communications for various postures," IEEE Transactions on Antennas and Propagation, vol. 57, no. 4, pp. 991-998, 2009.

[13] A. Sani and Y. Hao, "Modeling of path loss for ultrawide band body-centric wireless communications," in Proceedings of the International Conference on Electromagnetics in Advanced Applications (ICEAA'09), pp. 998-1001, Turin, Italy, September 2009.

[14] T. Zasowski, F. Althaus, M. Stager A, Wittneben, and G. Troster, "UWB for noninvasive wireless body area networks: channel measurements and results," in Proceedings of the IEEE Conference on Ultra Wideband Systems and Technologies, pp. 285-289, November 2003.

[15] A. Alomainy, Y. Hao, C. G. Parini, and P. S. Hall, "Comparison between two different antennas for UWB on-body propagation measurements," IEEE Antennas and Wireless Propagation Letters, vol. 4, no. 1, pp. 31-34, 2005.

[16] Q. H. Abbasi, A. Sani, A. Alomainy, and Y. Hao, "Arm movements effect on ultra wideband on-body propagation channels and radio systems," in Proceedings of the Loughborough Antennas and Propagation Conference (LAPC'09), pp. 261-264, Loughborough, UK, November 2009.

[17] A. Alomainy, Q. H. Abbasi, A. Sani, and Y. Hao, "System-level modelling of optimal ultra wideband body-centric wireless network," in Proceedings of the Asia Pacific Microwave Conference 2009 (APMC'09), pp. 2188-2191, Singapore, December 2009.

[18] A. Sani, G. Palikaras, A. Alomainy, and Y. Hao, "Time domain UWB radio channel characterisation for body-centric wireless communications in indoor environment," in Proceedings of the IET Seminar on Wideband and Ultrawideband Systems and Technologies: Evaluating Current Research and Development, no. 12352, November 2008.

[19] A. Sani, A. Alomainy, and Y. Hao, "Effect of the indoor environment on the UWB on-body radio propagation channel," in Proceedings of the 3rd European Conference on Antennas and Propagation (EuCAP'09), pp. 455-458, March 2009.

[20] A. Alomainy, A. Sani, A. Rahman, J. G. Santas, and Y. Hao, "Transient characteristics of wearable antennas and radio propagation channels for ultrawideband body-centric wireless communications," IEEE Transactions on Antennas and Propagation, vol. 57, no. 4, pp. 875-884, 2009.

[21] Plus Asset Tag, Reader and SFF Antenna Datasheet/Time Domain, 2009, http://www.timedomain.com/.

[22] K. P. Burnham and D. R. Anderson, Model Selection and Multimodel Inference: A Practical Information-Theoretic Approach, Springer, New York, NY, USA, 2002.

[23] S. S. Gassemzadeh, R. Jana, C. W. Rice, W. Turin, and V. Tarohk, "A statistical path loss model for in-home UWB channels," in IEEE Conference on Ultra Wideband Systems and Technologies, pp. 59-64, 2002.

[24] W. C. Y. Lee, Mobile Communications Engineering, Mc-GrawHill, New York, NY, USA, 1998. 

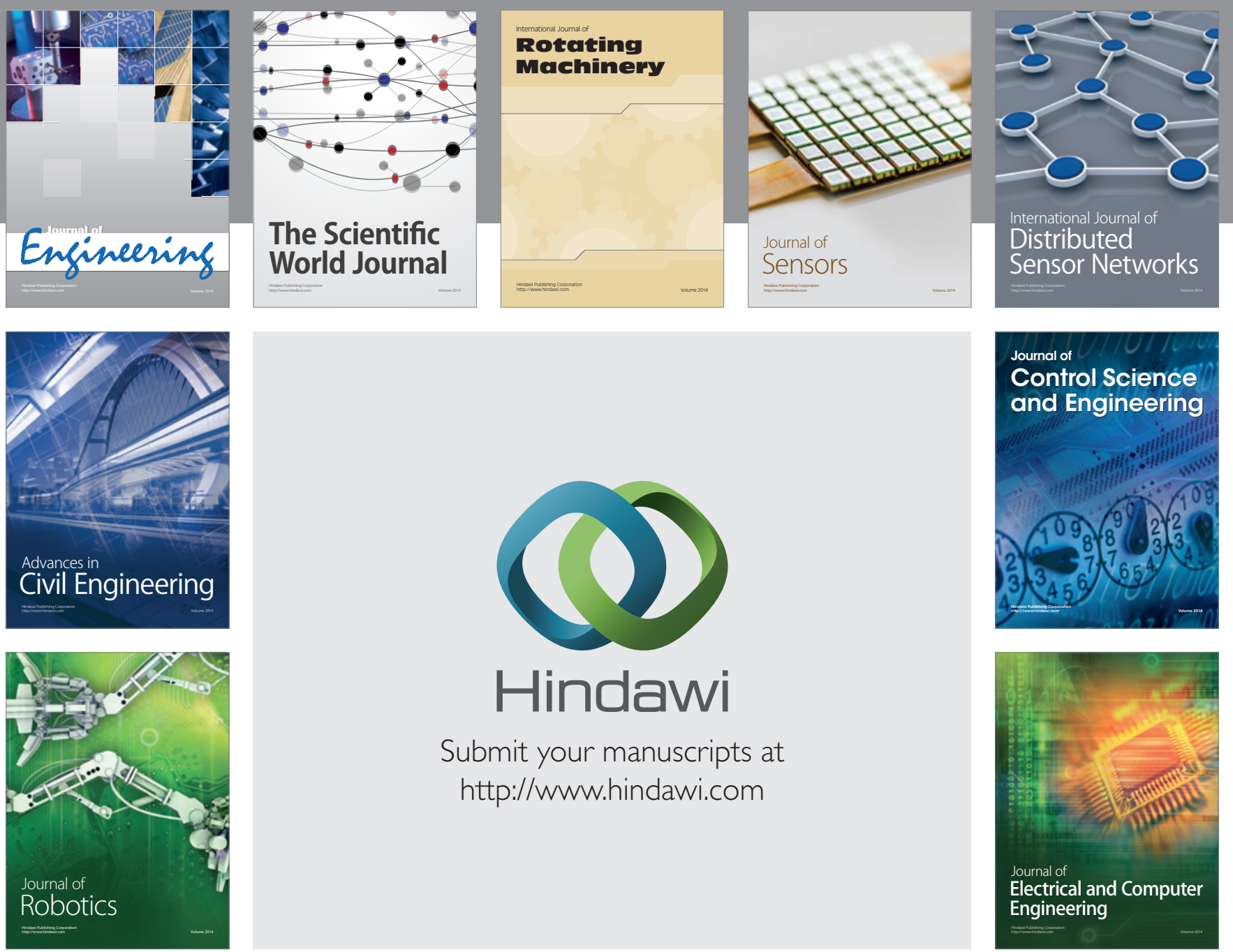

Submit your manuscripts at

http://www.hindawi.com
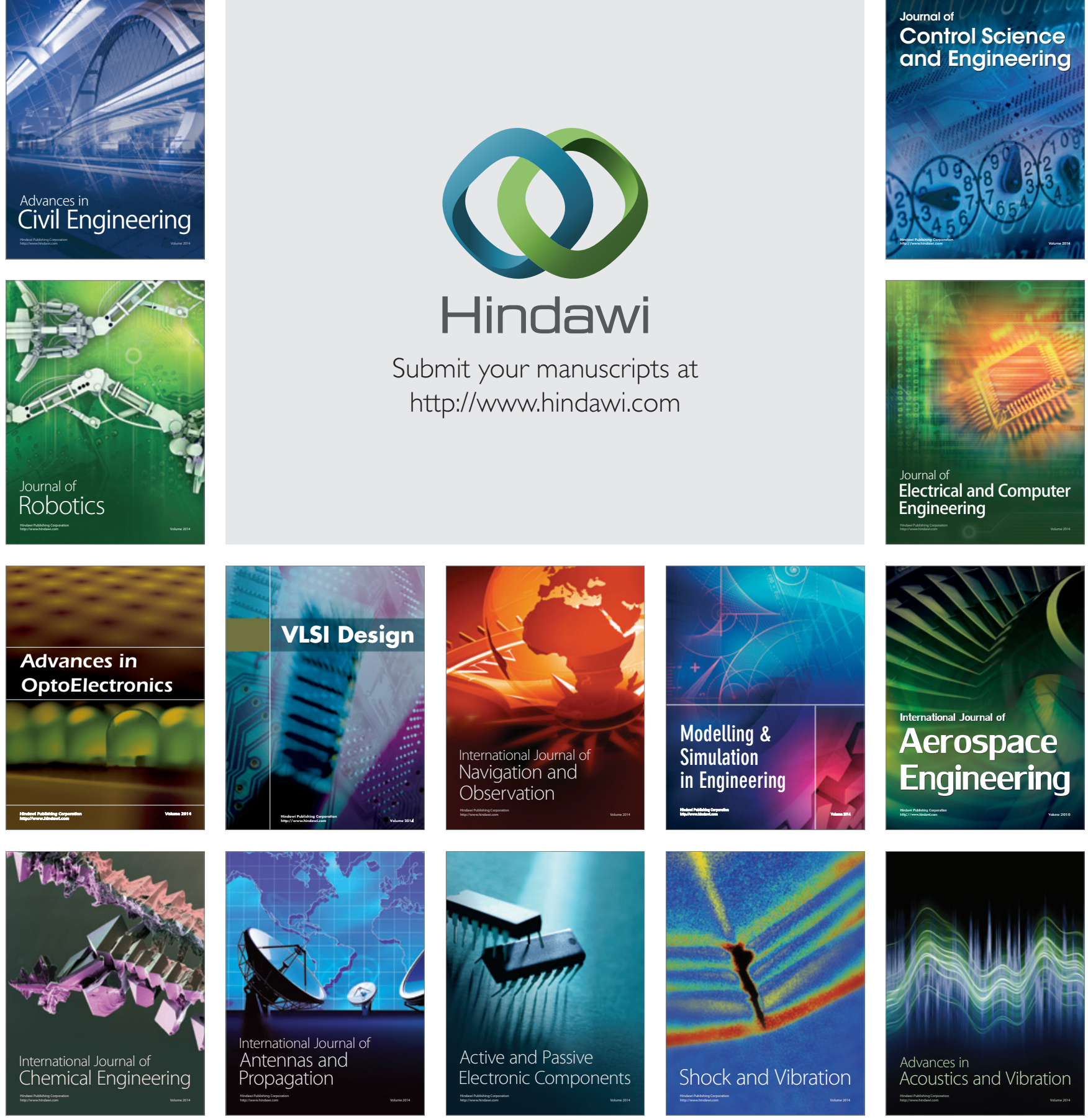